HEPHY-PUB 632/96

UWThPh-1995-23

March 1996

\title{
RELATIVISTIC COULOMB PROBLEM: ANALYTIC UPPER BOUNDS ON ENERGY LEVELS
}

\author{
Wolfgang LUCHA \\ Institut für Hochenergiephysik, \\ Österreichische Akademie der Wissenschaften, \\ Nikolsdorfergasse 18, A-1050 Wien, Austria

\section{Franz F. SCHÖBERL} \\ Institut für Theoretische Physik, \\ Universität Wien, \\ Boltzmanngasse 5, A-1090 Wien, Austria
}

\begin{abstract}
The spinless relativistic Coulomb problem is the bound-state problem for the spinless Salpeter equation (a standard approximation to the Bethe-Salpeter formalism as well as the most simple generalization of the nonrelativistic Schrödinger formalism towards incorporation of relativistic effects) with the Coulomb interaction potential (the static limit of the exchange of some massless bosons, as present in unbroken gauge theories). The nonlocal nature of the Hamiltonian encountered here, however, renders extremely difficult to obtain rigorous analytic statements on the corresponding solutions. In view of this rather unsatisfactory state of affairs, we derive (sets of) analytic upper bounds on the involved energy eigenvalues.
\end{abstract}




\section{Introduction: The Spinless Salpeter Equation}

Maybe one of the most straightforward generalizations of the standard nonrelativistic quantum theory towards the reconciliation with all the requirements imposed by special relativity is represented by describing the quantum systems under consideration by the well-known "spinless Salpeter equation." Consider a quantum system the dynamics of which is governed by a by assumption self-adjoint Hamiltonian $H$ of the form

$$
H=T+V,
$$

where $T$ denotes the square-root operator of the relativistic expression for the free (kinetic) energy of some particle of mass $m$ and momentum p,

$$
T \equiv \sqrt{\mathbf{p}^{2}+m^{2}},
$$

and $V=V(\mathbf{x})$ represents some arbitrary coordinate-dependent static interaction potential. The eigenvalue equation for this Hamiltonian $H$,

$$
H\left|\chi_{k}\right\rangle=E_{k}\left|\chi_{k}\right\rangle, \quad k=0,1,2, \ldots,
$$

for Hilbert-space eigenvectors $\left|\chi_{k}\right\rangle$ corresponding to energy eigenvalues

$$
E_{k} \equiv \frac{\left\langle\chi_{k}|H| \chi_{k}\right\rangle}{\left\langle\chi_{k} \mid \chi_{k}\right\rangle}
$$

is nothing else but the one-particle spinless Salpeter equation. Because of the nonlocality of this operator $H$, that is, more precisely, of either the kinetic-energy operator $T$ in configuration space or the interactionpotential operator $V$ in momentum space, it is hard to obtain analytic statements from this equation of motion. (For the "translation" of the equal-mass two-particle problem to the one-particle problem discussed at present, see Appendix A.)

\section{The Spinless Relativistic Coulomb Problem}

Of particular importance in all physics are the (spherically symmetric) central potentials, which depend only on the radial coordinate $r \equiv|\mathbf{x}|$. Among these, the most prominent one is the Coulomb potential $V_{\mathrm{C}}(r)$, which is parametrized by some (dimensionless) coupling constant $\alpha$ :

$$
V(\mathbf{x})=V_{\mathrm{C}}(r)=-\frac{\alpha}{r}, \quad \alpha>0
$$


The (semi-)relativistic Hamiltonian (四) with the Coulomb interaction potential $V_{\mathrm{C}}$ in (2) defines the "spinless relativistic Coulomb problem." In the past, the interest in this spinless relativistic Coulomb problem has undergone an eventful history. (For a rather comprehensive review, consult Ref. [1].) Let us merely sketch in the following some highlights.

First of all, from an examination [2] of the spectral properties of the operator (囵), (2) one may infer the existence of its Friedrichs extension up to the critical value

$$
\alpha_{\mathrm{c}}=\frac{2}{\pi}
$$

of the involved coupling constant $\alpha$ and read off a lower bound on the corresponding ground-state energy eigenvalue $E_{0}$, namely,

$$
E_{0} \geq m \sqrt{1-\left(\frac{\pi \alpha}{2}\right)^{2}} \quad \text { for } \alpha<\frac{2}{\pi},
$$

which, later on, has been slightly improved to [3]

$$
E_{0} \geq m \sqrt{\frac{1+\sqrt{1-4 \alpha^{2}}}{2}} \text { for } \alpha<\frac{1}{2} \text {. }
$$

The analytic solutions for the wave functions of those eigenstates $|\chi\rangle$ of the Hamiltonian (四), (8) which correspond to vanishing orbital angular momentum have been constructed [⿴囗十). The attempt in [田] to determine simultaneously the respective (set of) exact energy eigenvalues without actually solving this spinless Salpeter equation, however, failed [5, [0]. Therefore, as far as analytic statements about the relativistic Coulomb problem, in particular, its energy eigenvalues, are concerned, up to now one has to content oneself with a few series expansions of these energy eigenvalues $E_{k}$ in powers of the coupling constant $\alpha$ [7, 8], which then are, of course, only significant for a region of rather small values of $\alpha$.

\section{Analytic Upper Bounds on Energy Eigenvalues}

Without a closed form of all the energy eigenvalues $E_{k}$ of the spinless relativistic Coulomb problem at hand, it is highly desirable to have, at least, analytic expressions for upper bounds on these at one's disposal, in order to estimate the reliability of approximative solutions or series expansions.

The theoretical basis as well as the primary tool for the derivation of rigorous upper bounds on the eigenvalues of some self-adjoint operator 
is, beyond doubt, the so-called "min-max principle" [9]. An immediate consequence of this min-max principle is the following statement: Let $H$ be a self-adjoint operator that is bounded from below [as, according to Eq. (3)), evidently holds for the (semi-)relativistic Hamiltonian in (1) with a Coulomb-type interaction potential (2)]. Let $E_{k}, k=0,1,2, \ldots$, denote the eigenvalues of $H$, ordered according to $E_{0} \leq E_{1} \leq E_{2} \leq \ldots$. Let $D_{d}$ be some $d$-dimensional subspace of the domain of $H$. Then the $k$ th eigenvalue (counting multiplicity) of $H, E_{k}$, satisfies the inequality

$$
E_{k} \leq \sup _{\psi \in D_{k+1}} \frac{\langle\psi|H| \psi\rangle}{\langle\psi \mid \psi\rangle}, \quad k=0,1,2, \ldots
$$

A peculiarity of the (spinless relativistic) Coulomb problem is that there is only one dimensional parameter, namely, the particle mass $m$. As a consequence of this, for a vanishing particle mass, i. e., for $m=0$, the totality of eigenvalues $E_{k}$ of the Hamiltonian (四) with interaction potential (2) collapses, already on dimensional grounds, necessarily to $E_{k}=0$ for all $k=0,1,2, \ldots$. This fact is also clearly demonstrated by application of the "relativistic virial theorem" proven in Refs. [10, [1]]. Accordingly, in the course of an investigation of the spinless relativistic Coulomb problem, it is sufficient to focus one's interests to the special case of a nonvanishing particle mass $m$ :

$$
m>0 \text {. }
$$

In view of the above, when searching for bounds, our intention must be to avoid in some way or other the problematic square-root operator in order to deal with more manageable Hamiltonians.

\subsection{The "Schrödinger" bound}

From the positivity of the square of the obviously self-adjoint operator $T-m$,

$$
\begin{aligned}
0 & \leq(T-m)^{2} \\
& =T^{2}+m^{2}-2 m T \\
& =\mathbf{p}^{2}+2 m^{2}-2 m T
\end{aligned}
$$

one obtains, for the free (or kinetic) energy $T$, the operator inequality

$$
T \leq m+\frac{\mathbf{p}^{2}}{2 m}
$$


and thus, for the generic Hamiltonian $H$ in (四), the operator inequality

$$
H \leq H_{\mathrm{S}}
$$

where $H_{\mathrm{S}}$ denotes the "Schrödinger" Hamiltonian

$$
H_{\mathrm{S}}=m+\frac{\mathbf{p}^{2}}{2 m}+V .
$$

Applying to the energy eigenvalues $E_{k}$ of the Hamiltonian $H$ in Eq. (回) first the min-max principle in the form given by Eq. (困) and after that the operator inequality (5), we find

$$
\begin{aligned}
E_{k} & \equiv \frac{\left\langle\chi_{k}|H| \chi_{k}\right\rangle}{\left\langle\chi_{k} \mid \chi_{k}\right\rangle} \\
& \leq \sup _{\psi \in D_{k+1}} \frac{\langle\psi|H| \psi\rangle}{\langle\psi \mid \psi\rangle} \\
& \leq \sup _{\psi \in D_{k+1}} \frac{\left\langle\psi\left|H_{S}\right| \psi\right\rangle}{\langle\psi \mid \psi\rangle} .
\end{aligned}
$$

Now, let us assume that the $(k+1)$-dimensional subspace $D_{k+1}$ in this inequality is spanned by the first $k+1$ eigenvectors of the Schrödinger Hamiltonian $H_{\mathrm{S}}$, that is, by precisely those eigenvectors of $H_{\mathrm{S}}$ which correspond to the first $k+1$ energy eigenvalues $E_{\mathrm{S}, 0}, E_{\mathrm{S}, 1}, \ldots, E_{\mathrm{S}, k}$ when all eigenvalues of $H_{\mathrm{S}}$ are ordered according to $E_{\mathrm{S}, 0} \leq E_{\mathrm{S}, 1} \leq E_{\mathrm{S}, 2} \leq \ldots$. In this case, the right-hand side of the above inequality is nothing else but the $k$ th "Schrödinger" energy eigenvalue $E_{\mathrm{S}, k}$ :

$$
\sup _{\psi \in D_{k+1}} \frac{\left\langle\psi\left|H_{\mathrm{S}}\right| \psi\right\rangle}{\langle\psi \mid \psi\rangle}=E_{\mathrm{S}, k}
$$

Consequently, any single energy eigenvalue $E_{k}$ of the spinless Salpeter equation is bounded from above by its "Schrödinger" counterpart $E_{\mathrm{S}, k}$ :

$$
E_{k} \leq E_{\mathrm{S}, k}
$$

For the Coulomb potential (ㄱ) $)$, the energy eigenvalues required here are well known:

$$
E_{\mathrm{S}, n}=m\left(1-\frac{\alpha^{2}}{2 n^{2}}\right)
$$

where the total quantum number $n$ is given in terms of both radial and orbital angular momentum quantum numbers $n_{\mathrm{r}}$ and $\ell$, respectively, by

$$
n=n_{\mathrm{r}}+\ell+1, \quad n_{\mathrm{r}}=0,1,2, \ldots, \quad \ell=0,1,2, \ldots .
$$




\subsection{A "squared" bound}

One might be tempted to try to find an improvement of the bound (77) by considering the square of the Hamiltonian $H$, that is, the operator

$$
Q \equiv H^{2}=T^{2}+V^{2}+T V+V T .
$$

The eigenvalue equation for this squared Hamiltonian $Q$ will, of course, be solved by the same set of eigenvectors $\left|\chi_{k}\right\rangle$ as the one for the original Hamiltonian $H$ with, however, the squares of the corresponding energy eigenvalues $E_{k}$ as the eigenvalues of $Q$ :

$$
Q\left|\chi_{k}\right\rangle=E_{k}^{2}\left|\chi_{k}\right\rangle, \quad k=0,1,2, \ldots .
$$

From the positivity of the square of the self-adjoint operator $T-m-V$,

$$
\begin{aligned}
0 & \leq(T-m-V)^{2} \\
& =T^{2}+m^{2}+V^{2}-2 m T+2 m V-T V-V T,
\end{aligned}
$$

we obtain, with the help of the obvious relation

$$
0 \leq m \leq T
$$

for the anticommutator $T V+V T$ of kinetic energy $T$ and interaction potential $V$, which appears in the square $Q$ of our Hamiltonian $H$, the operator inequality

$$
\begin{aligned}
T V+V T & \leq T^{2}+m^{2}+V^{2}-2 m T+2 m V \\
& \equiv \mathbf{p}^{2}+2 m^{2}+V^{2}-2 m T+2 m V \\
& \leq \mathbf{p}^{2}+V^{2}+2 m V,
\end{aligned}
$$

which, when applied to the squared Hamiltonian $Q$, yields the operator inequality

$$
Q \leq R
$$

with the operator $R$ given by

$$
R \equiv 2 \mathbf{p}^{2}+m^{2}+2 V^{2}+2 m V .
$$

Following the line of argument as given in the preceding subsection, we might therefore conclude that the squares of the energy eigenvalues $E_{k}$ of the spinless relativistic Coulomb problem are bounded from above by the eigenvalues $\mathcal{E}_{\mathrm{R}, k}$ of the operator $R$ :

$$
E_{k}^{2} \leq \mathcal{E}_{\mathrm{R}, k}
$$


which entails

$$
E_{k} \leq \sqrt{\mathcal{E}_{\mathrm{R}, k}}
$$

Now, only for the Coulomb potential (2), this latter operator $R$ is of precisely the same structure as the Schrödinger Hamiltonian (5), with, however, some kind of "effective" orbital angular momentum quantum number $L$ which, for states of (genuine) orbital angular momentum $\ell$, has to be determined from the relation

$$
L(L+1)=\ell(\ell+1)+\alpha^{2},
$$

the solution of which reads

$$
L=\frac{\sqrt{1+4\left[\ell(\ell+1)+\alpha^{2}\right]}-1}{2}, \quad \ell=0,1,2, \ldots .
$$

In this case, the eigenvalues $\mathcal{E}_{\mathrm{R}}$ of the operator $R$ may be easily written down:

$$
\mathcal{E}_{\mathrm{R}, N}=m^{2}\left(1-\frac{\alpha^{2}}{2 N^{2}}\right),
$$

with the "effective" total quantum number

$$
N=n_{\mathrm{r}}+L+1, \quad n_{\mathrm{r}}=0,1,2, \ldots .
$$

Unfortunately, it is easy to convince oneself that the "square" bounds (9) obtained in this way lie above and are thus worse than the previous Schrödinger bounds.

\subsection{A variational bound}

For all practical purposes, the most efficient manner for the min-max principle to come into play is in form of the "Rayleigh-Ritz variational technique." Let us illustrate this fact just for the ground-state energy eigenvalue $E_{0}$. For $k=0$, the relation (困) simplifies to

$$
E_{0} \leq \frac{\langle\psi|H| \psi\rangle}{\langle\psi \mid \psi\rangle}
$$

That is, the ground-state energy $E_{0}$ is clearly less than or equal to any expectation value of the considered Hamiltonian, $H$. The above upper bound may, of course, be optimized by determining the smallest of all these expectation values, at least in some chosen Hilbert-space sector. Consequently, there is a simple recipe for the derivation of (sometimes excellent!) exact upper bounds on this ground-state energy eigenvalue: 
minimize the encountered expectation values of the Hamiltonian under consideration,

$$
\frac{\left\langle\psi_{\lambda}|H| \psi_{\lambda}\right\rangle}{\left\langle\psi_{\lambda} \mid \psi_{\lambda}\right\rangle}
$$

with respect to a suitably chosen set of Hilbert-space trial vectors $\left|\psi_{\lambda}\right\rangle$, which are distinguished from each other by some variational parameter $\lambda$.

We apply this prescription to our (semi-)relativistic Hamiltonian $H$. However, in order to make life easy, we immediately take advantage of a simple inequality for the expectation values of a self-adjoint operator, like our kinetic energy $T$, with respect to arbitrary Hilbert-space states $|\psi\rangle$ in the domain of this operator:

$$
\frac{|\langle\psi|T| \psi\rangle|}{\langle\psi \mid \psi\rangle} \leq \sqrt{\frac{\left\langle\psi\left|T^{2}\right| \psi\right\rangle}{\langle\psi \mid \psi\rangle}} .
$$

Employing this inequality, we are able to circumvent the (troublesome) expectation values of the square-root operator of the kinetic energy $T$ (which, of course, may be always evaluated numerically; see Ref. [回):

$$
\begin{aligned}
E_{0} & \leq \frac{\left\langle\psi_{\lambda}|H| \psi_{\lambda}\right\rangle}{\left\langle\psi_{\lambda} \mid \psi_{\lambda}\right\rangle} \\
& =\frac{\left\langle\psi_{\lambda}|T+V| \psi_{\lambda}\right\rangle}{\left\langle\psi_{\lambda} \mid \psi_{\lambda}\right\rangle} \\
& \leq \sqrt{\frac{\left\langle\psi_{\lambda}\left|T^{2}\right| \psi_{\lambda}\right\rangle}{\left\langle\psi_{\lambda} \mid \psi_{\lambda}\right\rangle}+\frac{\left\langle\psi_{\lambda}|V| \psi_{\lambda}\right\rangle}{\left\langle\psi_{\lambda} \mid \psi_{\lambda}\right\rangle}} \\
& \equiv \sqrt{\frac{\left\langle\psi_{\lambda}\left|\mathbf{p}^{2}\right| \psi_{\lambda}\right\rangle}{\left\langle\psi_{\lambda} \mid \psi_{\lambda}\right\rangle}+m^{2}}+\frac{\left\langle\psi_{\lambda}|V| \psi_{\lambda}\right\rangle}{\left\langle\psi_{\lambda} \mid \psi_{\lambda}\right\rangle}
\end{aligned}
$$

For the Coulomb potential (2), the most reasonable choice of trial vectors is obviously one for which the coordinate-space representation $\psi_{\lambda}(\mathbf{x})$ of the trial vectors $\left|\psi_{\lambda}\right\rangle$ for vanishing radial and orbital angular momentum quantum numbers is given by hydrogen-like trial functions:

$$
\psi_{\lambda}(\mathbf{x})=\exp (-\lambda r), \quad \lambda>0 .
$$

For these trial functions, the computation of the expectation values in the above inequality yields [6]

$$
E_{0} \leq \sqrt{\lambda^{2}+m^{2}}-\alpha \lambda
$$


Determining the minimum of this latter set of upper bounds, we arrive at [6]

$$
E_{0} \leq E_{\text {var }, 0},
$$

with the variational upper bound for the ground-state energy level $E_{0}$ of the spinless relativistic Coulomb problem given by

$$
E_{\mathrm{var}, 0} \equiv m \sqrt{1-\alpha^{2}} \text {. }
$$

This variational bound, $E_{\mathrm{var}, 0}$, is lower and thus better than the former Schrödinger bound on the ground-state energy level,

$$
E_{\mathrm{S}, 0}=m\left(1-\frac{\alpha^{2}}{2}\right)
$$

which may be obtained from Eq. (8) for $n_{\mathrm{r}}=\ell=0$, implying thereby $n=1$ :

$$
E_{\mathrm{var}, 0}<E_{\mathrm{S}, 0} \text { for } \alpha \neq 0 .
$$

Consequently, the variational technique entails indeed improved upper bounds on the energy levels as compared to the Schrödinger estimates.

\subsection{A straightforward generalization}

Our variational upper bound ([0) for the ground-state energy level $E_{0}$ can be very easily re-derived and simultaneously extended to arbitrary levels of excitation by a generalization of the considerations presented in Subsection 3.1. Introducing an arbitrary real parameter $\mu$ (with the dimension of mass), we make use of the positivity of the square of the obviously self-adjoint operator $T-\mu$,

$$
\begin{aligned}
0 & \leq(T-\mu)^{2} \\
& =T^{2}+\mu^{2}-2 \mu T \\
& =\mathbf{p}^{2}+m^{2}+\mu^{2}-2 \mu T,
\end{aligned}
$$

in order to find, for the kinetic energy $T$, a set of operator inequalities,

$$
T \leq \frac{\mathbf{p}^{2}+m^{2}+\mu^{2}}{2 \mu} \text { for all } \mu>0
$$

and, consequently, for the (semi-)relativistic Hamiltonian $H$ in Eq. (四), the set of operator inequalities

$$
H \leq \widehat{H}_{\mathrm{S}}(\mu) \text { for all } \mu>0
$$


with the Schrödinger-type Hamiltonian $\widehat{H}_{\mathrm{S}}(\mu)$ given by

$$
\widehat{H}_{\mathrm{S}}(\mu)=\frac{\mathbf{p}^{2}+m^{2}+\mu^{2}}{2 \mu}+V .
$$

Now, mimicking the line of argument given in Subsection 3.1 involving the min-max principle, we conclude that the set of energy eigenvalues $E_{k}, k=0,1,2, \ldots$, of the Hamiltonian $H$, if again ordered according to $E_{0} \leq E_{1} \leq E_{2} \leq \ldots$, is bounded from above by the (corresponding) set of energy eigenvalues $\widehat{E}_{\mathrm{S}, k}(\mu)$ of this Schrödinger Hamiltonian $\widehat{H}_{\mathrm{S}}(\mu)$, if again similarly ordered according to $\widehat{E}_{\mathrm{S}, 0}(\mu) \leq \widehat{E}_{\mathrm{S}, 1}(\mu) \leq \widehat{E}_{\mathrm{S}, 2}(\mu) \leq \ldots$,

$$
E_{k} \leq \widehat{E}_{\mathrm{S}, k}(\mu) \text { for all } \mu>0,
$$

and, consequently, also by the minimum of all these energy eigenvalues:

$$
E_{k} \leq \min _{\mu>0} \widehat{E}_{\mathrm{S}, k}(\mu)
$$

For the Coulomb potential (2) $)$, these energy eigenvalues $\widehat{E}_{\mathrm{S}, n}(\mu)$ are given by

$$
\widehat{E}_{\mathrm{S}, n}(\mu)=\frac{1}{2 \mu}\left[m^{2}+\mu^{2}\left(1-\frac{\alpha^{2}}{n^{2}}\right)\right],
$$

with precisely the same total quantum number $n$ as before. Minimizing this latter expression with respect to the parameter $\mu$, we finally obtain

$$
\min _{\mu>0} \widehat{E}_{\mathrm{S}, n}(\mu)=m \sqrt{1-\frac{\alpha^{2}}{n^{2}}} .
$$

For any value of the total quantum number $n$, these bounds definitely improve the Schrödinger bounds (8):

$$
\min _{\mu>0} \widehat{E}_{\mathrm{S}, n}(\mu)<E_{\mathrm{S}, n} \quad \text { for } \alpha \neq 0 .
$$

Clearly, for $\mu=m$, we immediately recover the Schrödinger approach of Subsection 3.1.

\section{Summary}

Horrified by the insight that the discrete spectrum of the Hamiltonian consisting of just the relativistic kinetic energy and the static Coulomb interaction potential is still not known exactly, we derived by different but elementary methods at least a few complete sets of analytic upper 
bounds on the respective energy eigenvalues. In every individual case, the basic idea was to derive an operator inequality for this Hamiltonian which guarantees that the expectation values of this Hamiltonian are bounded from above by the expectation values of some other operator which no longer involves the (problematic) square-root operator of the relativistic kinetic energy, and to "embed" this operator inequality into the well-known min-max principle for the eigenvalues of a self-adjoint operator bounded from below. It should be no great surprise that just that method which employs some variational procedure yields the best of these bounds.

\section{Acknowledgements}

We would like to thank B. Baumgartner, H. Grosse, H. Narnhofer, W. Thirring, and J. Yngvason for a lot of helpful discussions.

\section{A Equivalence of One-Particle and (Equal-Mass) Two-Particle Scenarios}

The two relativistic Coulombic Hamiltonians for one- and two-particle problem,

and

$$
H^{(1)}=\sqrt{\mathbf{p}^{2}+m^{2}}-\frac{\alpha}{r}, \quad r \equiv|\mathbf{x}|
$$

$$
H^{(2)}=2 \sqrt{\mathbf{P}^{2}+M^{2}}-\frac{\kappa}{R}, \quad R \equiv|\mathbf{X}|,
$$

respectively, may be equated with the help of a scale transformation as follows. Relate the employed phase-space variables, $(\mathbf{x}, \mathbf{p})$ and $(\mathbf{X}, \mathbf{P})$, respectively, by some (in general arbitrary) scale factor $\lambda$ according to

$$
\begin{aligned}
& \mathbf{p}=\lambda \mathbf{P}, \\
& \mathbf{x}=\frac{\mathbf{X}}{\lambda},
\end{aligned}
$$

which preserves their fundamental commutation relations:

$$
[\mathbf{x}, \mathbf{p}]=[\mathbf{X}, \mathbf{P}] \text {. }
$$

Fix this scale factor, $\lambda$, to the particular value $\lambda=2$ and identify both the mass and the Coulomb coupling strength parameters according to

$$
\begin{aligned}
m & =2 M \\
\alpha & =\frac{\kappa}{2} .
\end{aligned}
$$


You will end up with

$$
H^{(1)}=H^{(2)} \text {. }
$$

\section{References}

[1] W. Lucha and F. F. Schöberl, All Around the Spinless Salpeter Equation, in: Proceedings of the International Conference on Quark Confinement and the Hadron Spectrum (Como, Italy, June 1994), eds. N. Brambilla and G. M. Prosperi (World Scientific, River Edge, N. J., 1995) p. 100.

[2] I. W. Herbst, Commun. Math. Phys. 53 (1977) 285; 55 (1977) 316 (addendum).

[3] A. Martin and S. M. Roy, Phys. Lett. B 233 (1989) 407.

[4] B. Durand and L. Durand, Phys. Rev. D 28 (1983) 396; 50 (1994) 6662 (erratum).

[5] G. Hardekopf and J. Sucher, Phys. Rev. A 31 (1985) 2020.

[6] W. Lucha and F. F. Schöberl, Vienna preprint HEPHY-PUB 596/94 (1994) (unpublished);

W. Lucha and F. F. Schöberl, Phys. Rev. D 50 (1994) 5443.

[7] A. Le Yaouanc, L. Oliver, and J.-C. Raynal, Ann. Phys. (N. Y.) 239 (1995) 243.

[8] N. Brambilla and A. Vairo, Phys. Lett. B 359 (1995) 133.

[9] M. Reed and B. Simon, Methods of Modern Mathematical Physics IV: Analysis of Operators (Academic Press, New York, 1978) Section XIII.1.

[10] W. Lucha and F. F. Schöberl, Phys. Rev. Lett. 64 (1990) 2733.

[11] W. Lucha and F. F. Schöberl, Mod. Phys. Lett. A5 (1990) 2473. 